ISSN 0103-9954

\title{
EFECTO DEL RELIEVE Y LA INTENSIDAD DE TRÁNSITO EN COSECHA SOBRE LA DINÁMICA DE LA REGENERACIÓN NATURAL DE UN BOSQUE NEOTROPICAL EN MISIONES, ARGENTINA
}

\section{SLOPE AND TRAFFIC INTENSITY EFFECTS ON THE DYNAMICS OF NATURAL REGENERATION IN NEOTROPICAL FOREST IN MISIONES, ARGENTINA}

\section{Nardia Bulfe $^{1} \quad$ Liliana Rivero $^{2} \quad$ Mariela Teresczcuch $^{3} \quad$ Patricio Mac Donagh $^{4}$ \\ RESUMEN}

Con el objetivo de evaluar la dinámica de la regeneración natural establecida en las vías de saca y la influencia del terreno y la intensidad de tránsito sobre ellas, en el año 2002 se instalaron 32 pares de parcelas de $4 \mathrm{~m}^{2}$ cada una en la Reserva de Uso Múltiple Guaraní, en Misiones, Argentina. En el área fueron aplicadas diferentes técnicas de aprovechamiento en el año 1999. Las parcelas se instalaron en sitios con pendiente $(7 \%$ a $30 \%)$ y baja declividad $(<7 \%)$ con dos intensidades de tránsito en cada una de ellas, en ambientes transitados (vías de saca) y no transitados (bajo dosel). Fueron considerados todos los individuos mayores a $30 \mathrm{~cm}$ de altura y menores a $10 \mathrm{~cm}$ de DAP. Se calcularon los diferentes parámetros para los años 2002 y 2004. Fueron relevados un total de 20.547 renuevos por hectárea, en donde el 61 \% fue registrado bajo el dosel. La densidad en las vías de saca fue estadísticamente inferior a la encontrada en la primera medición ( $\mathrm{n}=32 ; \mathrm{t}=31,0 ; \mathrm{p}=$ 0,00067). Los mayores incrementos en altura se dieron en las vías de saca. Los mayores incrementos diamétricos promedios fueron registrados en las vías de saca en sitios con pendiente. La diversidad fue mayor en sitios sin pendiente. La similaridad entre pares de parcelas disminuye en sitios con pendiente y a mayores intensidades de tránsito.

Palabras clave: regeneración natural; aprovechamiento; vías de saca; sucesión.

\section{ABSTRACT}

An experiment was carried out to evaluate the dynamics of the natural regeneration settled down in the skid trails and the influence of the slope and the traffic intensity on them, comparing three and five years after logging. The forest belongs to Selva Paranaense, type of forest in the Yabotí Biosphere Reserve, and in the Guarani Reserve of the Misiones National University, in Misiones, Argentina. The harvesting was carried out in 1999, through the comparison of two methods: Reduced Impact Logging and Conventional Logging. Thirty two couples of plots of four squared meters settled were established in places with high slope (7\% to $30 \%$ ) and low slope ( $<7 \%$ ) with two traffic intensities in each one of them (skid trails) and not trafficked area (below canopy). All the bigger individuals were considered to 30 centimeters high and smaller to 10 centimeters of D.B.H. All parameters were calculated for the years 2002 and 2004. It was raised a total of 20547 seedlings by hectare where $61 \%$ were registered under canopy. The abundance in the skid trails was statistically lower in the first mensuration $(\mathrm{n}=32 ; \mathrm{t}=31.0 ; \mathrm{p}=0.00067)$. The biggest increments in height were given in the skid trails. The biggest mean diametric increases were registered in the skid trails in places with slope. The diversity was bigger in places without slope. The similarity among some plots diminishes in places with slope and in higher traffic intensities.

Keyword: natural regeneration; logging; skid trails; succession.

\section{INTRODUCCION}

El Bosque Atlántico es uno de los ecosistemas más diversos del mundo, motivo por el cual su estudio y comprensión se torna complejo. Este tipo de bosque se encuentra en tres países, Argentina, Brasil y Paraguay. Si bien Argentina posee la mayor superficie remanente de estos bosques, los mismos presentan un

1. Ingeniera Forestal, Becaria de Posgrado, Universidade Federal do Paraná, Rua Lothário Meissner, 632, Jardim Botânico, CEP 80210-160, Curitiba (PR). nardia_bulfe@yahoo.com.ar

2. Ingeniera Forestal, Becaria de Posgrado, Universidad Nacional de Misiones, Bertoni 124, Eldorado, Misiones Argentina. li_rivero@yahoo.com.ar

3. Ingeniera Forestal, Becaria de Posgrado, Universidad Nacional de Misiones, Bertoni 124, Eldorado, Misiones Argentina. mteresczcuch@facfor.unam.edu.ar

4. Ingeniero Forestal, M.Sc., Universidad Nacional de Misiones, Bertoni 124, Eldorado, Misiones Argentina. mdonagh@facfor.unam.edu.ar

Recebido para publicação em 28/02/2006 e aceito em 20/04/2007. 
alto grado de intervención.

Diferentes mecanismos relacionados a la conservación de los recursos naturales resultaron poco exitosos, siendo reflejo de ello la degradación sucesiva de los bosques en las últimas décadas. Uno de los principales problemas es el escaso conocimiento sobre la dinámica de estos bosques (DI BITETTI et al., 2003).

La dinámica de una comunidad forestal depende de diversos factores que pueden actuar en conjunto, entre ellos uno de los más importantes es la composición específica. A su vez las variables ambientales, dadas en un determinado lugar, son las que controlan la composición específica de dicho ambiente.

De acuerdo a la exploración de los recursos, existen dos grandes grupos de especies, heliófitas y esciófitas. Debido a la predominancia de las heliófitas en las primeras etapas de la sucesión, las tasas de cambio en esta etapa son mayores; dichas especies presentan vida corta, con un rango entre 7 - 25 años, mayormente entre 10 - 20 años (CORLETT, 1995).

Estas especies dominan el área en los primeros años de sucesión creando condiciones óptimas para las especies clímax, las cuales aparecen bajo la sombra de las primeras, reemplazándolas. De esta manera la composición y estructura del bosque convergería en algún punto con la del bosque circundarte (GUARIGUATA y OSTERTAG, 2001; CORLETT, 1995). Según estos autores, el bosque original y el tipo de suelo, cobran importancia en la sucesión, así como también el tipo e intensidad del disturbio y el banco de semillas dentro del bosque. Fredericksen y Mostacedo (2000) determinaron en un bosque de Bolivia, que el establecimiento y crecimiento de la regeneración natural incrementa con el aumento del disturbio en el suelo, atribuyendo principalmente a dos especies intolerantes a la sombra las cuales podrían estar siendo beneficiadas por el incremento en luz debido a la apertura del dosel.

Autores como Uhl y Vieira (1989); Jackson et al. (2002), Toledo et al. (2001); Pinard et al. (1996); y Todd y Pariona (2001), han llegado a la conclusión de que los caminos y las operaciones de extracción se tornan en actividades críticas en el aprovechamiento forestal, influyendo en la dinámica de la regeneración natural de las especies a causa de la erosión y compactación de suelos que estos provocan. Es por ello que el aprovechamiento de Mínimo Impacto podría ser considerado como una herramienta factible en el manejo de los bosques. Existen datos que demuestran la disminución de la extensión total de caminos en un aprovechamiento, esto puede lograrse mediante una mejor planificación de los mismos (DYKSTRA, 1997; SIST et al., 1998; BULFE et al., 2005).

Schorn (2005) en la evaluación de diferentes estadios sucesionales en una Floresta Ombrófila Densa en Brasil (SC), encontró que la regeneración natural, en el estadio inicial de dicha sucesión, el 50,3\% de los individuos fueron del grupo ecológico "pioneras", con 18,7\% de "climax" exigentes en luz. Por otro lado, el estadio más avanzado de sucesión de la composición florística era compuesta en un $83 \%$ por especies "climax".

En la extracción maderera se utilizan maquinarias de gran porte que en sucesivas pasadas compactan el suelo y remueven la capa superficial. Según la intensidad, se destruye la vegetación existente y se impide o retrasa la germinación de semillas existentes en el banco del suelo. Si bien la intensidad de tránsito es un factor importante cuando se analiza el impacto del aprovechamiento, la situación del terreno podría tener una relación más estrecha sobre el impacto en la vegetación (BULFE et al., 2003/2004). En situaciones con pendiente se hace más difícil el tránsito de la maquinaria, por lo que el mismo se concentra en pocos lugares, intensificando el disturbio en los mismos.

Es por ello que el presente trabajo tiene como objetivo contribuir al conocimiento de parte de la dinámica de estos bosques, evaluando la dinámica de la regeneración natural establecida en las vías de extracción maderera en función de factores como la pendiente y la intensidad de tránsito.

\section{MATERIALES Y METODOS}

\section{Área de estudio}

El estudio se llevó a cabo en la Reserva Forestal de Uso Múltiple Guaraní (UNaM), ubicada dentro de la Reserva de Biosfera Yabotí, en la provincia de Misiones, Argentina. Posee una superficie total de 5.343 hectáreas. Está ubicada en el departamento Guaraní, municipio El Soberbio, a los 26 57’ de latitud sur y 
54 $4^{\circ} 15^{\prime}$ de longitud oeste. El paisaje es serrano con algunas pendientes escarpadas. El punto más alto es de 574 m.s.n.m. en el sector sur.

El clima de la región corresponde, según Köeppen al tipo Cfa, que es un clima macrotérmico, constantemente húmedo y subtropical; con una precipitación media anual de $1800 \mathrm{~mm}$ y una temperatura media anual de $21^{\circ} \mathrm{C}$.

Los suelos se presentan como complejos cartográficos, conocidos localmente como 6a y 6b, y de acuerdo a la taxonomía del USDA, los órdenes más importantes son: Haplortoxes, Kaniudults, Kandihunults, Umbracualfs, Hapludalfs, Kandiudalfs, Argiudoles, Hapludoles, Haplumbrepts, Distrocrepts, Udortents (PHAR et al., 1997).

De acuerdo a la clasificación establecida por Cabrera y Willink (1980), el área se sitúa en la región fitogeográfica denominada "Provincia Paranaense", Distrito de las Selvas; este tipo de bosque es también denominado como bosque estacional semidecidual.

La vegetación predominante del Bosque Atlántico del Alto Paraná es el bosque subtropical semidecíduo. En esta región se destacan las comunidades del palo rosa, cedro, guatambú, incienso, laurel y palmito (DI BITETTI et al., 2003).

Tressens y Revilla (1997), encontraron en el predio Guaraní 114 especies arbóreas siendo éstas de gran porte, medianos y bajos; las mismas se encontraban distribuidas en 92 géneros representadas por 42 familias. Por otra parte en un ensayo en el mismo predio, Mac Donagh et al. (en prensa) hallaron 102 especies de árboles con un promedio de 46 especies por hectárea. La densidad media fue de 277 árboles por hectárea.

\section{Procedimiento metodológico}

\section{Descripción del ensayo}

En un área de 100 hectáreas seleccionadas dentro del área descripta se aplicaron diferentes modalidades de aprovechamiento:

a) Tratamiento de cosecha de impacto reducido (CIR), cuyo fundamento fue aplicar criterios de sustentabilidad en la selección de los árboles a cortar. En función de esto se estableció el recorrido de la maquinaria dentro de la parcela, la ubicación de las vías de saca y las planchadas. Como consecuencia se establecieron las intensidades de tránsito permisibles para cada sector del terreno (9 parcelas).

b) Tratamiento de cosecha comercial (CC) donde el obrajero (contratista de maquinaria) debió seleccionar los individuos a cortar, y también estableció sus recorridos y planchadas dentro de cada parcela.

Quedando 3 parcelas como Control (PT), en donde no se cortaron árboles, y no se realizó ningún tránsito con maquinaria (3 parcelas).

Las parcelas tenían cada una 200 x 200 m, con una parcela central de 1 ha donde se concentraron las mediciones de estructura y regeneración arbórea. En ellas se identificaron y midieron todos los árboles $>10$ cm de diámetro a la altura del pecho (DAP) (Figura 1).

La cosecha se realizó entre junio y julio de 1999. Los árboles fueron cortados empleando una motosierra Stihl 070. Luego el árbol era despuntado y transformado en trozas de tamaño variable siendo "amarradas" con cable de acero, y arrastradas hasta planchada por un skidder de 10 toneladas de peso total, rodado 18,4 x $34 \mathrm{~m}$. En 18 parcelas de 4 ha cada una, fueron relevados todos los ejemplares arbóreos y palmas de más de $10 \mathrm{~cm}$ de DAP; así como también la regeneración natural existente en cada parcela, antes y después del aprovechamiento. Durante la cosecha, se relevaron todos los parámetros de producción, y la intensidad de tránsito. 


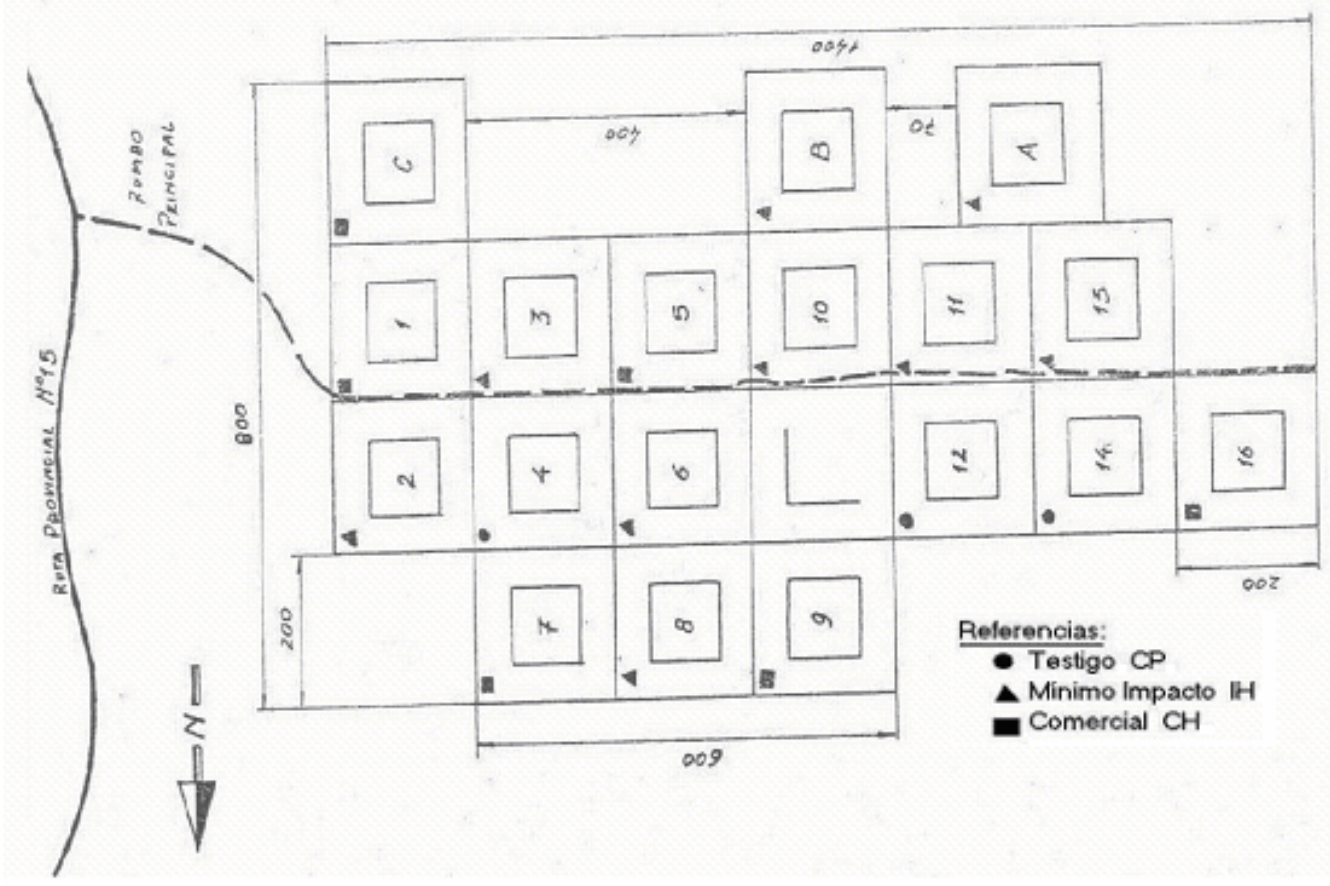

FIGURA 1: Representación esquemática del ensayo general en la Reserva de Uso Múltiple Guaraní en Misiones, Argentina.

FIGURE 1: Field map showing treatment and plots distributions in Guarani Reservation Area, Misiones, Argentine.

A fin de lograr el objetivo planteado en este trabajo, se seleccionaron cuatro parcelas de 4 hectáreas en función de la similitud en composición florística y estructura (GARIBALDI et al., 2002), que fueron cosechadas tal como describió Mac Donagh (2000). De estas cuatro parcelas se identificaron dos en terreno con pendiente ( $7 \%$ a $30 \%$ ) y dos en terreno de baja declividad $(<7 \%)$. A su vez se consideraron los distintos rangos de intensidades de tránsito que resultaron del número de pasadas con el tractor por la vía de saca, de manera tal de que se establecieron parcelas en un primer rango (Intensidad 1: de 2 a 6 pasadas) y en un segundo rango para las mayores intensidades (Intensidad 2: más de 12 pasadas). Una vez seleccionadas las áreas se procedió a la instalación de cuatro parcelas pareadas, de $4 \mathrm{~m}^{2}$ cada una en las vías de saca y en sitios no transitados (bajo dosel) (FIGURA 2). Se instaló un total de 32 pares de parcelas, en donde se identificaron, marcaron y midieron todos los individuos con más de 30 centímetros $(\mathrm{cm})$ de altura y menores a $10 \mathrm{~cm}$ de DAP. Se midió la altura total de los individuos en metros (m) y el diámetro a la altura del cuello en milímetros (DAC) Esta medición se repitió luego de dos años de la primera. 


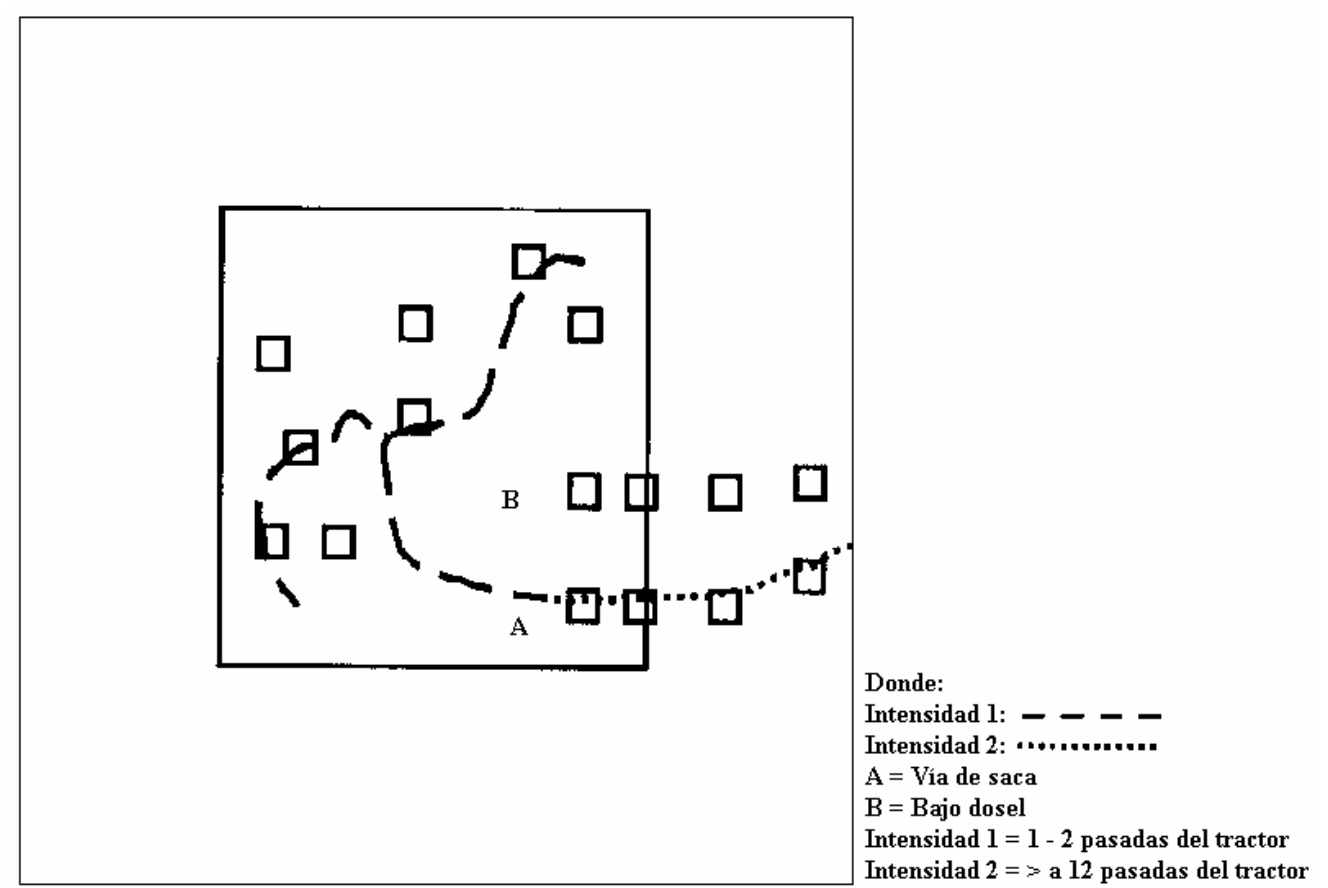

FIGURA 2: Representación esquemática de los pares de parcelas de regeneración natural establecidas en las vías de saca y bajo dosel.

FIGURE 2: Natural regeneration schematic design of pairs of sub-plots among skid trails and understory.

\section{Evaluación fitosociológica}

Los datos obtenidos en campo fueron procesados en el programa FITOPAC I (SHEPHERD, 1988). Para la determinación de los diferentes parámetros estructurales se utilizaron los datos correspondientes a los años 2002 y 2004. Se determinaron los parámetros fitosociológicos de frecuencia, dominancia, densidad, índice de Valor de Importancia y los índices de diversidad de Shannon $\left(\mathrm{H}_{1}\right)$ (HOSOKAWA, 1986) y de similitud de Jaccard (MATEUCCI y COLMA, 1982). Fueron también calculados los incrementos diamétricos e incrementos en altura para el período de medición. El análisis de los datos consistió en la comparación de las variables de la regeneración natural durante el periodo de estudio (2002-2004).

\section{Análisis estadístico}

Los datos fueron comparados a través de la estadística no paramétrica. El test utilizado fue Wilcoxon para muestras pareadas, con un nivel de $\alpha=0,05$, realizando comparaciones entre años de medición (CANTATORE DE FRANK, 1983). También fueron determinados los parámetros estadísticos (desvío estándar, promedio y error estándar) para los incrementos en diámetro y en altura. Los incrementos en altura y diámetro fueron representados usando gráficos de tipo "Box Plot`s"

\section{RESULTADOS Y DISCUSION}

Fueron relevados un total de 526 renovales en todo el ensayo, los cuales pertenecieron a 54 especies distribuidas en 28 familias botánicas. El 39 \% de los renovales fue registrado en las vías de saca mientras que el $61 \%$ restante se estableció en condiciones de bajo dosel, en donde solamente 21 especies del total fueron comunes para ambos ambientes.

Los registros de densidad en las vías de saca en el año 2004 fueron estadísticamente inferiores a los encontrados en el año 2002 ( $n=32 ; t=31,0 ; p=0,00067)$, mientras que bajo dosel el comportamiento fue inverso, no siendo esta diferencia estadísticamente significativa $(n=32 ; \mathrm{t}=117,5 ; \mathrm{p}=0,353)$.

Éstos valores se muestran en la tabla 1 donde se observa que existe un comportamiento diferenciado de acuerdo a la pendiente del terreno. En terrenos con pendiente, y a mayores intensidades de tránsito, la densidad aumenta coincidiendo ésto con lo determinado por Fredericksen y Mostacedo (2000). En sitios con pendiente el parámetro densidad es siempre mayor que en los sitios sin pendiente para el año 2004. Esto 
podría estar relacionado a varios factores, entre ellos la orientación de la pendiente, que podría ser en este caso favorable para el establecimiento de los individuos. Otro factor de importancia es el número de pasadas de la maquinaria, que en sitios con pendiente tiende a alcanzar un número máximo de pasadas mayor que en sitios sin pendiente. Esto podría estar provocando una mayor apertura en el bosque y una mayor remoción a nivel del suelo. Los mencionados efectos en parte se explican por el tipo de maquinaria empleada durante la extracción, que al ser relativamente pequeña, el tránsito en sitios con pendiente se tornó más dificultoso, teniendo que dividir la carga o muchas veces hacer varios intentos para avanzar. Por otra parte, estas situaciones suelen asociarse con suelos rocosos y someros (MAC DONAGH et al., 2001). También es de importancia mencionar las estrategias de las plantas para su establecimiento en el bosque. La mayoría de las especies pioneras presentan latencia y una alta diseminación, motivo por el cual son persistentes y dominantes en el banco de semillas aguardando la disponibilidad del recurso limitante para su germinación, en este caso la luz (BACH, 2000).

TABLA 1: Parámetros estructurales para cada situación bajo estudio (valores promedios por hectárea), en la Reserva Forestal de Uso Múltiple Guaraní, Misiones, Argentina.

TABLE 1: Structural parameters for each situation (averages by hectare) in Guarani Reservation Area, Misiones, Argentine.

\begin{tabular}{l|c|c|c|c|c|c}
\hline Parametros & \multicolumn{2}{|c|}{ Densidad } & \multicolumn{2}{c|}{ Dominancia } & \multicolumn{2}{c}{ Altura } \\
\hline Año de medición & 2002 & 2004 & 2002 & 2004 & 2002 & 2004 \\
\hline A1CP & 18750 & 16562,5 & 16,20 & 25,76 & 2,05 & 2,68 \\
A2CP & 23750 & 22812,5 & 17,30 & 18,60 & 2,69 & 1,93 \\
B1CP & 27812,5 & 33125 & 6,25 & 9,00 & 1,31 & 1,33 \\
B2CP & 31562,5 & 38125 & 4,14 & 8,31 & 0,81 & 0,85 \\
A1SP & 21562,5 & 11875 & 22,28 & 17,59 & 3,08 & 3,53 \\
A2SP & 19687,5 & 16250 & 8,77 & 6,42 & 1,76 & 0,99 \\
B1SP & 22187,5 & 13125 & 8,81 & 10,32 & 0,93 & 0,59 \\
B2SP & 13437,5 & 11875 & 2,85 & 17,59 & 0,58 & 0,70 \\
\hline
\end{tabular}

Donde: A: Vías de Saca; B: Bajo Dosel; 1: Intensidad de tránsito 1 (2-6 pasadas); 2: Intensidad de tránsito 2 (> 12 pasadas); SP: Sin Pendiente; CP: Con Pendiente.

Al comparar ambos años de medición se hace notar que está ocurriendo la sucesión, debido a la disminución de la densidad promedio y al aumento de la dominancia en la mayoría de las situaciones bajo estudio. El incremento de la dominancia producido bajo dosel en la intensidad de tránsito 2 en sitios sin pendiente podría estar relacionado a la mayor densidad de Urera baccifera L. (Gaudich) y Trema micrantha L. (Blume), ya que estas especies presentan un incremento diamétrico alto por ser consideradas de comportamiento heliófito; esto coincide con lo mencionado por Corlett (1995) donde menciona la importancia de las especies pioneras en las primeras etapas de la sucesión, debido a que estas otorgan una mayor dinámica en el bosque.

En cuanto a la altura promedio estimada para cada situación se observa que los mayores valores, en ambos años de medición, se dieron en las vías de saca principalmente en la intensidad de tránsito menor (Intensidad de tránsito 1) (Tabla 1). Esto estaría indicando la existencia de una menor apertura a causa de un número menor de pasadas de la maquinaria dada en las menores intensidades de tránsito, promoviendo que los renovales tengan un mayor crecimiento en altura por la búsqueda de luz en un estrato superior.

El incremento promedio anual de la altura de los renovales fue mayor en las vías de saca en sitios con pendiente. Dependiendo de la intensidad de tránsito, estos demostraron ser mayores a intensidades menores (Figura 1). 


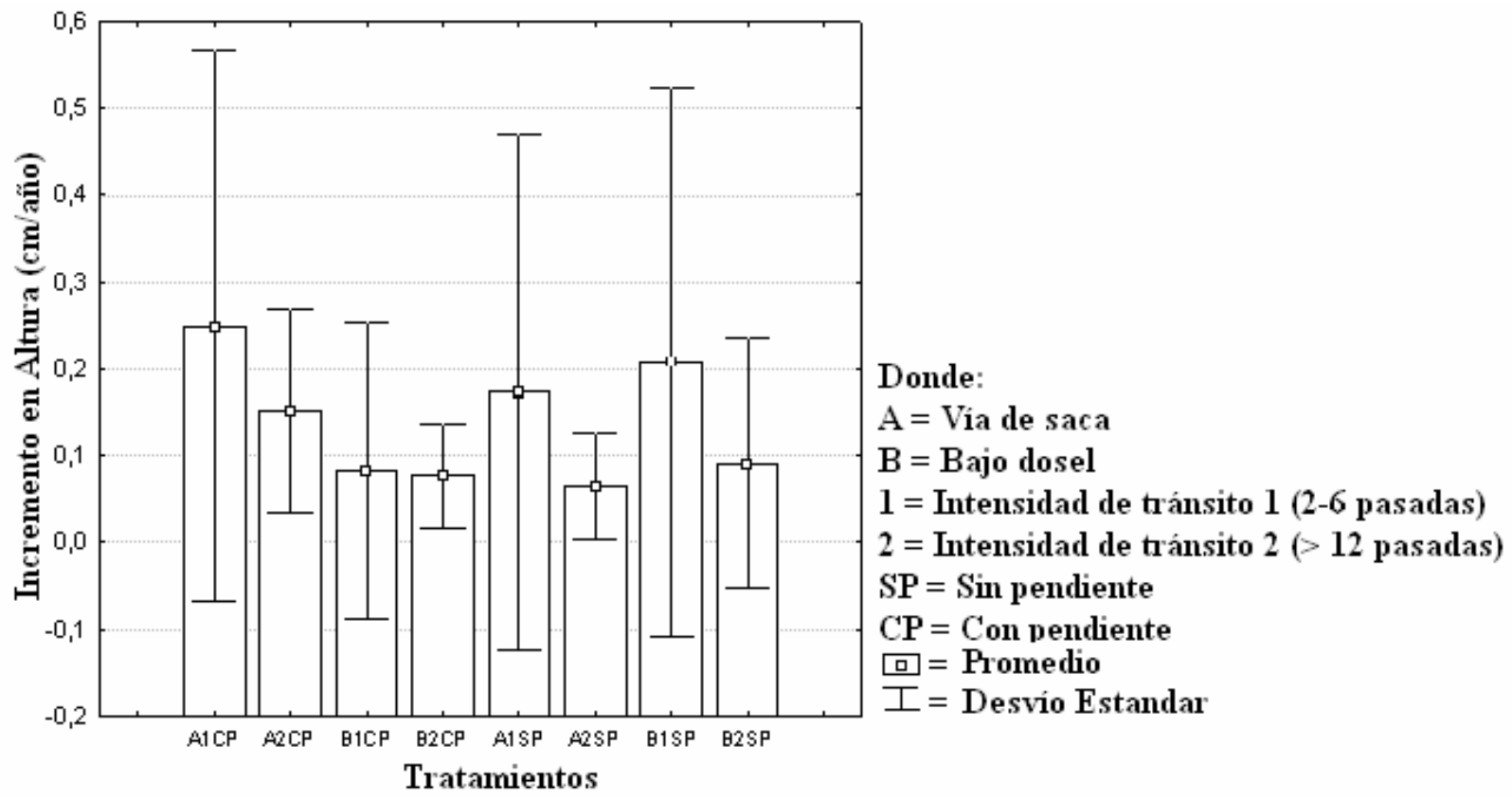

FIGURA 1: Incremento en altura de renuevos (cm/año) para el periodo 2002-2004 en cada tratamiento, en la Reserva Forestal de Uso Múltiple Guaraní, Misiones, Argentina.

FIGURE 1: Increase height (cm/year) from 2002 to 2004 in each situation in Guarani Reservation Area, Misiones, Argentine.

Los incrementos diamétricos en cada situación bajo estudio se pueden apreciar en la figura 2. Se observa que los mayores incrementos promedios fueron registrados en las vías de saca en sitios con pendiente.

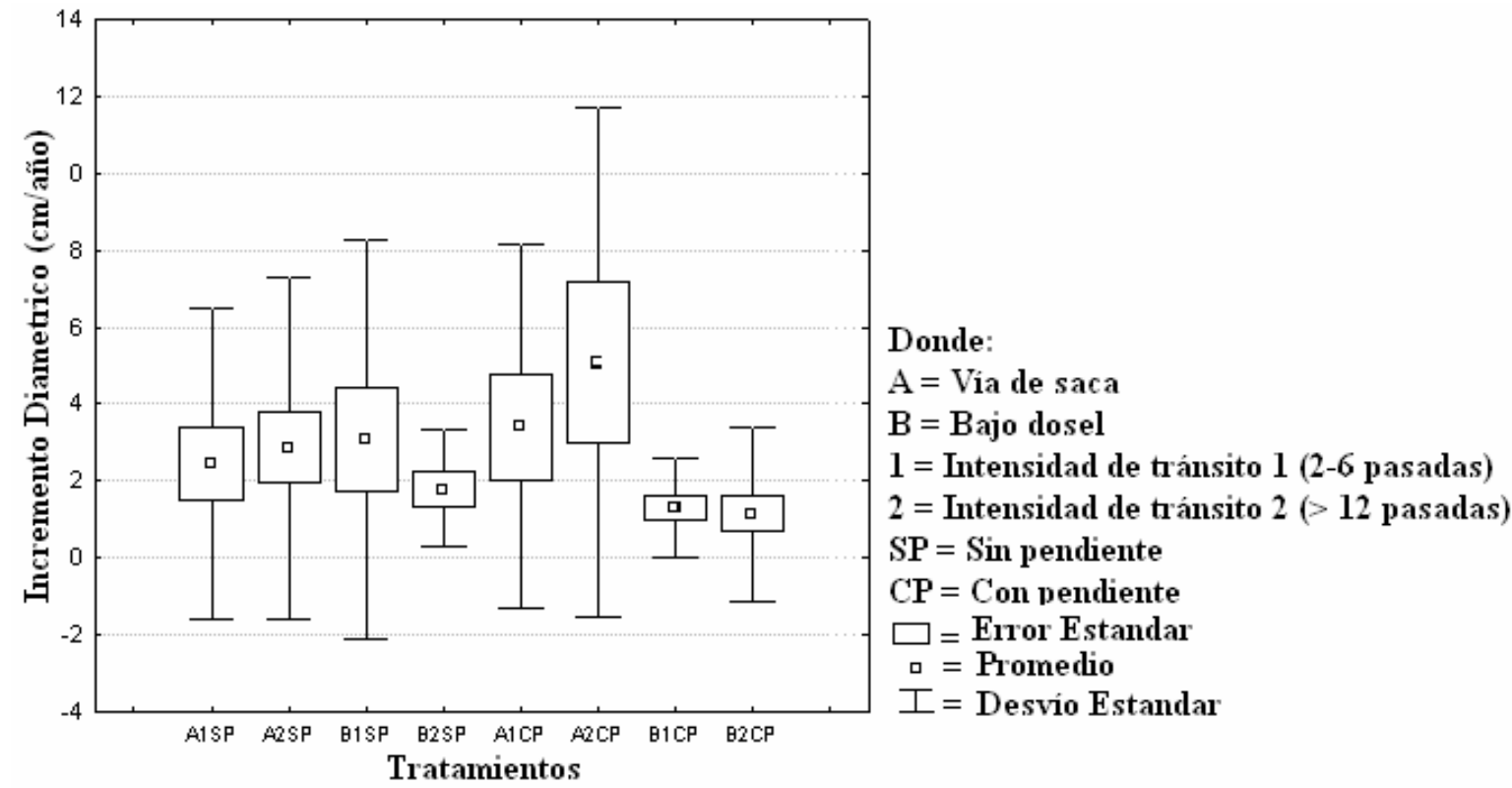

FIGURA 2: Incremento diamétrico promedio (cm) por tratamiento, en la Reserva Forestal de Uso Múltiple Guaraní, Misiones, Argentina.

FIGURE 2: Increase diameter average $(\mathrm{cm})$ for each situation in Guarani Reservation Area, Misiones, Argentine.

En situaciones bajo dosel los promedios de incrementos diamétricos son menores y presentan desvíos menores en la mayoría de los casos. Esto es un reflejo de la composición de especies en cada ambiente. Aquellos ambientes limitados en cuanto a la entrada de luz están compuestos, en su mayoría por 
individuos de especies tolerantes a la sombra, mientras que los sitios abiertos son caracterizados por la presencia de individuos de especies de carácter heliófilo, siendo cada grupo ecológico responsable por la dinámica hallada en ambos ambientes.

La diversidad sigue teniendo el mismo comportamiento que el observado por Bulfe et al. (2003). Los sitios sin pendiente siguen presentando los mayores índices de diversidad en el año 2004.

Al comparar los índices entre los años 2002-2004 se observa que por lo general en las vías de saca este índice aumenta, sucediendo lo contrario para situaciones bajo dosel (Tabla 2).

TABLA 2: Indices de diversidad Shannon para cada situación de estudio, en la Reserva Forestal de Uso Múltiple Guaraní, Misiones, Argentina.

TABLE 2: Index of Shannon diversity in Guarani Reservation Area, Misiones, Argentine.

\begin{tabular}{l|c|c|c|c|c|r|r|c}
\hline Años & A1CP & A2CP & B1CP & B2CP & A1SP & A2SP & B1SP & B2SP \\
\hline Año 2002 & 1,81 & 1,5 & 2,61 & 2,66 & 2,64 & 3,09 & 2,03 & 1,74 \\
Año 2004 & 2,04 & 1,65 & 2,31 & 2,51 & 2,74 & 3,03 & 2,06 & 1,61 \\
\hline
\end{tabular}

Donde: A: Vía de saca; B: Bajo dosel; 1: Intensidad de tránsito 1 (2-6 pasadas); 2: Intensidad de tránsito 2 (> 12 pasadas); CP: Con pendiente; SP: Sin pendiente.

Este aumento de la diversidad en las vías de saca está en relación al número de especies, ya que en este ambiente el ingreso de nuevas especies tuvo una importancia mayor que en el ambiente de bajo dosel.

En cuanto a la similaridad entre los ambientes (Tabla 3) cabe mencionar que los rangos de valores determinados para el año 2004 presentan el mismo comportamiento a los hallados por Bulfe et al. (2003), en donde la similaridad disminuye en sitios con pendiente y con mayor intensidad de tránsito.

TABLA 3: Rangos de índices de similaridad de Jaccard en el año 2004, en la Reserva Forestal de Uso Múltiple Guaraní, Misiones, Argentina.

TABLE 3: Ranges of index of Jaccard similarity in 2004 in Guarani Reservation Area, Misiones, Argentine.

\begin{tabular}{l|c|c|c|c}
\hline Años & A1-B1 SP & A2-B2 SP & A1-B1 CP & A2-B2 CP \\
\hline 2002 & $0-0,5$ & $0-0,25$ & $0-0,2$ & $0-0,08$ \\
2004 & $0-0,22$ & $0-0,20$ & $0-0,22$ & $0-0,17$ \\
\hline
\end{tabular}

Donde: A: Vía de saca; B: Bajo dosel; 1: Intensidad de tránsito 1 (2-6 pasadas); 2: Intensidad de tránsito 2 (> 12 pasadas); SP: Sin pendiente; CP: Con pendiente.

Es importante destacar la disminución en el rango de similaridad en sitios sin pendiente, así como también el aumento en dicho índice en sitios con pendiente cuando comparados con los rangos del año 2002.

Un factor importante que influye en la regeneración natural es el sotobosque predominante en el sitio. En el área de estudio se observó que en sitios con pendiente existe una predominancia de sotobosque de latifoliadas, dando de esta manera mejores condiciones de luminosidad. Sin embargo, en sitios sin pendiente las bambúceas se tornan predominantes, inhibiendo de esta manera la regeneración de especies mediante la competencia por unos de los principales recursos, la luz.

\section{CONCLUSIONES}

Los parámetros determinados en los diferentes ambientes reflejan el comportamiento de las diferentes composiciones específicas en cada uno de ellos. Las vías de saca se encuentran aún en la primera etapa de la sucesión, en donde las especies allí establecidas están generando el microclima adecuado para se produzca el reemplazo de especies heliófilas por aquellas esciófilas.

Tanto el relieve como la intensidad de tránsito tienen una estrecha relación con la regeneración natural de especies arbóreas. Los incrementos en altura y DAP se vieron influenciados por ambos factores. El incremento en DAP fue mayor a mayores intensidades de tránsito mientras que los incrementos en altura fueron mayores a intensidades de tránsito menores y en sitios con pendiente, influenciados por la mayor competencia por luz en estos ambientes.

La dinámica de la regeneración natural establecida en las vías de saca hace pensar que el impacto de la cosecha sobre la regeneración arbórea, está más asociado al tipo de terreno que a la intensidad de tránsito. 
Efecto del relieve y la intensidad de tránsito en cosecha sobre la dinámica de la regeneración natural ...237

\section{AGRADECIMIENTOS}

Al Center for Internacional Forestry Research por co-financiar el presente proyecto.

\section{REFERENCIA BIBLIOGRAFICA}

BULFE, N.; RIVERO, L.; EIBL, B. et al. Efectos del arrastre de madera en cosecha selectiva, sobre la regeneración natural de un bosque neotropical en Misiones, Argentina. In: JORNADAS TÉCNICAS FORESTALES Y AMBIENTALES, 10., 2003, Eldorado. Anales... Eldorado: FCF-INTA, 2003. 1 CD-ROM.

BULFE, N.; RIVERO, L.; EIBL, B. et al. Efectos del relieve y la intensidad de tránsito sobre la regeneración natural de un bosque aprovechado en Misiones, Argentina. In: REUNIÓN BINACIONAL DE ECOLOGÍA, 2, 2004, Mendoza; REUNIÓN ARGENTINA DE ECOLOGÍA, 21., 2004, Mendoza; REUNIÓN DE LA SOCIEDAD DE ECOLOGÍA DE CHILE, 11, 2004, Mendoza. Anales... Mendoza : Sociedad de Ecología, 2004. p.244.

BULFE, N.; RIVERO, L.; JUAREZ, G. et al. Efectos de dos sistemas de aprovechamiento sobre la regeneración natural en vías de saca de un bosque nativo en Misiones Argentina. In: CONGRESO FORESTAL ARGENTINO Y LATINOAMERICANO, 3., 2005, Corrientes. Anales... Corrientes: Asociación Forestal Argentina, 2005. 1 CD-ROM.

CABRERA, A. L.; WILLINK, A. Biogeografía de América Latina. Washington: OEA, 1980. 117p. (Monografía, 13).

CANTATORE DE FRANK, N. Manual de estadística aplicada. Buenos Aires : Hemisferio Sur, 1983. Tomo II, p. 57-71.

CORLETT, R. T. Tropical secondary forest. Progress in Physical Geography, v. 19, n. 2, p. 159-172, 1995.

DI BITETTI, M.; PLACCI, G.; DIETZ, L. Una visión de biodiversidad para la ecoregión del Bosque Atlántico del Alto Paraná: diseño de un paisaje para la conservación de la biodiversidad y prioridades para las acciones de conservación. Washington, DC : World Wildlife Fund., 2003.

DYKSTRA, D. Aprovechamiento de impacto reducido: convirtiendo los resultados de la Investigación en prácticas de campo. In: SIMPOSIO INTERNACIONAL SOBRE POSIBILIDADES DE MANEJO FORESTAL SOSTENIBLE EN AMÉRICA TROPICAL, 1997, Santa Cruz de la Sierra. Memoria... Santa Cruz : BOLFOR-CIFOR-IUFRO, 1998. p.183-189.

FREDERICKSEN T. S.; MOSTACEDO B. Regeneration of timber species following selection logging in Bolivian tropical dry forest. Forest Ecology and Management, Amsterdam, v. 131, p. 47-55, 2000.

GARIBALDI, J.; RIVERO, L.; MAC DONAGH, P. Estructura y distribución espacial de especies arbóreas y su asociación a variables ambientales en un bosque de la reserva de biosfera Yabotí. JORNADAS TÉCNICAS FORESTALES Y AMBIENTALES, 9., 2002, Eldorado. Anais... Eldorado: FCF-INTA, 2002. 1 CD-ROM.

GUARIGUATA, M.; OSTERTAG, R. Neotropical secondary forest succession: changes in structural and functional characteristics. Forest Ecology and Management, Amsterdam, v. 148, p. 185-206, 2001.

HOSOKAWA, R. Manejo e economía de florestas. Roma: FAO: Programa das Nações Unidas para o Desenvolvimento, 1986. 125p.

JACKSON, S.; FREDERICKSEN, T.; MALCOLM, J. Area disturbed and residual stand damage following logging in a Bolivian tropical forest. Forest Ecology and Management, Amsterdam, v. 166, p. 271-283, 2002.

MAC DONAGH, P. Forest harvesting impacts evaluation on forest ecosystems: final report for phase I. Misiones: FCF, UNAM, CIFOR, 2000.

MAC DONAGH, P.; GARIBALDI, J.; ALVEZ, M. et al. Evaluación del impacto de la cosecha selectiva de madera en bosques subtropicales en Misiones, Argentina. In: CONGRESO LATINOAMERICANO DE INGENIERÍA RURAL. 2001, Guanajuato. Anales... Guanajuato, 2001. 1 CD-ROM.

MAC DONAGH, P.; GARIBALDI, J.; RIVERO, L. et al. A comparison between conventional and reduced impact timber harvesting on damage, mortality and recruitment of a neotropical forest. En prensa.

MATEUCCI, S. D.; COLMA, A. Metodología para el estudio de la vegetación. Coro : Universidad Nacional Experimental Francisco Miranda, 1982. 169p.

NICHOLS, J. D.; WAGNER, R. W.; AGYEMAN V. K. et al. Influence of artificial gaps in Tropical Forest on Survival, Growth, and Phytolyma lata attack on Milicia excelsa. Forest Ecology and Management, Amsterdam, v. 110, p. 353362, 1998.

PHAR, N.; FERNANDEZ, R.; O’LERY, H. et al. Descripción del suelo de la Reserva de Uso Múltiple Guaraní. Misiones : Instituto Subtropical de Investigaciones Forestales : Facultad de Ciencias Forestales, Universidad Nacional de 
Misiones. 1997. 40p.

PINARD, M.; DAVIDSON, D.; HOWLETT, B. Site conditions limit pioneer tree recruitment after logging of dioterocarp forest in Sabah, Malasia. Biotropica, Washington, v. 28, n. 1, p. 2-12, 1996.

PLANA BACH, E. Introducción a la ecología y dinámica del bosque tropical. Bloque II: Gestión forestal y agroforestería en los trópicos. Barcelona : Centro Tecnológico Forestal de Catalunya, 2000. 11 p. (Curso sobre gestión y conservación de bosques tropicales)

SCHORN, L. A. Estrutura e dinâmica de estágios sucessionais de uma floresta ombrófila densa em Blumenau, Santa Catarina. 2005. 119f. Tese (Doutorado em Engenharia Florestal) - Setor de Ciências Agrárias, Universidade Federal do Paraná, Curitiba.

SHEPHERD, G. J. Fitopac 1: versão preliminar-Software computacional e manual do usuário. Campinas: Departamento de Botânica da UNICAMP, 1988. 8p.

SIST, P.; DYKSTRA, D.; FIMBEL, R. Reduced-Impact Logging Guidelines for Lowland and Hill Dipterocarp Forest in Indonesia. Center for International Forestry Research, 1998. 19 p. (Occasional Paper; n. 15)

TODD, F.; PARIONA, W. Efectos de las alteraciones causadas por "Skidders” en la regeneración de árboles comerciales en claros de aprovechamiento en un bosque tropical de Bolivia. Santa Cruz : BOLFOR, 2001. 17p. (Proyecto de manejo Forestal Sostenible).

TOLEDO, M.; TODD, F.; LICONA, J. et al. Impacto del aprovechamiento forestal en la flora de un bosque semideciduo pluviestacional de Bolivia. Santa Cruz : BOLFOR, 2001. 23p. (Proyecto de manejo Forestal Sostenible).

TRESSENS, S.; REVILLA, V. Riqueza florística de un bosque nativo de 5000 Has. conducido racionalmente en la provincia de Misiones. In: CONGRESO ARGENTINO Y LATINOAMERICANO, 2.,1997, Posadas. Anales: bosque nativo y protección ambiental. Misiones, 1997. 1 CD-ROM.

UHL, C.; VIEIRA, I. Ecological impacts of selective logging in the Brazilian Amazonan: a case study from the Paragominas region of the state of Pará. Biotropica, Washington, v. 21, n. 2, p. 98-106, 1989. 